\title{
BMJ Open FOREST protocol: a qualitative study exploring health and sexuality of transmasculine individuals in France
}

\author{
Xavier Mabire (D) ,,2 Suzanne Robin-Radier, ${ }^{2,3}$ OUTrans NGO, ${ }^{3}$ Dulce Ferraz (D) ,1,2,4 \\ Marie Preau (D) ,2 FOREST research group
}

To cite: Mabire X, RobinRadier S, OUTrans NGO, et al. FOREST protocol: a qualitative study exploring health and sexuality of transmasculine individuals in France. BMJ Open 2021;11:e052748. doi:10.1136/ bmjopen-2021-052748

- Prepublication history and additional supplemental material for this paper are available online. To view these files, please visit the journal online (http://dx.doi.org/10.1136/ bmjopen-2021-052748).

Received 27 April 2021 Accepted 03 November 2021

\section{Check for updates}

(c) Author(s) (or their employer(s)) 2021. Re-use permitted under CC BY-NC. No commercial re-use. See rights and permissions. Published by BMJ.

${ }^{1}$ PHASE (Psychology of Health, Aging and Sport Examination), University of Lausanne Faculty of Social and Political Science, Lausanne, Vaud, Switzerland UMR 1296 "Radiations: Défense, Santé,

Environnement", Université Lyon 2, Lyon, Auvergne-Rhône-Alpes, France

${ }^{3}$ Community-Based

Organization, OUTrans NGO,

Paris, Île-de-France, France

${ }^{4}$ Escola FIOCRUZ de Governo,

Fundação Oswaldo Cruz,

Brasília, Brazil

Correspondence to

Dr Xavier Mabire;

xavier.mabire@unil.ch

\section{ABSTRACT}

Introduction While current research on sexuality and health often explores sexual behaviours among heterosexual and gay cisgender individuals, little is known about the sexualities of transgender people, especially transmasculine people. When data are available, sexual health is often reduced to risk exposure, not considering in detail social context and determinants that could contribute to a more comprehensive approach, such as general health, class, race, exposure to violence or social representations. Recognising this gap, identified in both national (French) and international scientific literature, this study aims to explore the sexual health of transmasculine people, employing an intersectional approach and considering both positive and negative health determinants.

Methods This 2-year research based in the disciplinary field of social psychology, with a gender perspective, and will apply qualitative methods. We adopt a communitybased research approach, integrating one university and one community-based organisation in the coordination of the study. In a triangulation perspective, two rounds of semistructured interviews will be performed with key informants (medical practitioners, communitybased support services workers, etc) and with people self-identifying as transmasculine. Focus groups will complement data collection.

Ethics and dissemination FOREST protocol was approved by the Comité d'Évaluation Éthique (CEEI) de I'Institut National de la Santé Et de la Recherche Médicale (CEEI/International Review Board 00003888). The research adopts the principles of open science, and findings will be published assuring participants' confidentiality. Informative flyers and videos will be elaborated to communicate study findings to participants, stakeholders and the transcommunities at large, and data will be stored in lasting archives.

\section{INTRODUCTION}

\section{Trans-specific scarce data}

Transgender people are those whose assigned sex at birth differs from their current gender identity or expression. Currently, scientific data on transgender people and their health status are scarce. ${ }^{1}$ Often, available data are based on extrapolations from other sexual or gender minorities in an undifferentiated

\section{Strengths and limitations of this study}

- The research mobilises a community-based research approach, articulating one university and one community-based organisation as study coordinators.

- It focuses on transmasculine people specifically, with an intersectional perspective.

- It employs an exploratory method through qualitative analysis to investigate intimacy and sexuality of this gender minority.

- The study offers innovative restitution methods for participants and the community, including lasting archives and social media dissemination.

- Qualitative data collection, nevertheless, implies limits in generalisation and representativeness of the sample.

manner, ${ }^{23}$ leading to artificial aggregations between populations that are not homogeneous. Among transgender people, all the light remains to be shed on transmasculine people's situation which is particularly undocumented.

The literature shows that to increase scientific knowledge and inform interventions towards transmasculine persons it is necessary to develop specific, disaggregated studies, preferably applying community-based approaches, both high or low-income and middle-income countries. ${ }^{4}$ Our research will focus specifically on this population group, exploring the functions of and social representations associated with sexuality in relation to HIV and other sexually transmitted infections (STIs) in transmasculine people.

\section{Transgender overall and sexual health}

Transmasculine people face many vulnerabilities regarding health. Data on the subject are either non-existent or incomplete, while being of serious concern. In short, the need for research among this population is well identified in the national and international context but unmet yet. ${ }^{1}$ To account for all the 
potential vulnerability factors and explore how being a transmasculine person affects health status and can lead to exposure to HIV and STIs, it seems essential to take into account social inequalities in health. This posture may help to better understand barriers, but also to identify possible levers to health.

\section{Overall health}

One of the first limitations in describing the overall health of transgender people is that there is a problem in defining gender categories and therefore in measuring them. ${ }^{15}$ The historically widespread practice of aggregating transgender people with other sexual or gender minorities including men who have sex with men renders their potential specificities invisible.

To date, there is no cohort, or representative sample that allows for robust cross-sectional or longitudinal measures of transgender health status. However, among the available data, a meta-analysis ${ }^{1}$ identifies six indicators of transgender health: general health status, mental health, violence and victimisation, stigma and discrimination, substance use, and sexual and reproductive health. This offers a starting point to explore the various dimensions of the psychosocial context that impacts health and sexuality of transmasculine people.

\section{Sexual health, HIV and STIs}

It has been observed that the sexual quality of life is little studied among transgender people. A few studies approach this subject in the frame of gender-affirmative surgeries ${ }^{1}$; otherwise, sexual health is studied primarily through the prism of HIV. Even concerning these two topics, the available data predominantly involve transgender women.

Various exposure factors contribute to the dynamics of the HIV epidemic: gender, migratory trajectory, social status, education level, practice of sex work, configuration of emotional and sexual networks, among others. ${ }^{6}$ Among transgender women, the available epidemiological data indicate an alarming situation, with global HIV prevalence estimated at $19 \% .^{2}$ The numbers may vary greatly from region to region, but in any case, the prevalence remains particularly high compared with the general population. HIV, thus, represents a substantial threat to the health of transgender women and, potentially, of transgender men.

Data concerning this last group are still very complicated to obtain, even though vulnerability to HIV and other STIs in transmasculine individuals has been identified as a priority area for research. ${ }^{7-9}$ The absence of data complicates the adaptation of health education and promotion programmes or the planning of sexual health strategies. Concerning sexuality, recent studies have highlighted the link between body dysphoria experience (before and after engaging in a transition path) and sexuality. ${ }^{10}$ If engaging in transition, some effects on sexuality have been reported, including 're-negotiating previous 'norms' and 'establishing identity'. ${ }^{11}$ If engaging sexual activities, transmasculine and nonbinary individuals indicate a wide repertoire of body use. ${ }^{12}$ Sexual satisfaction, ${ }^{13}$ or dissatisfaction ${ }^{14}$ independently of engaging in medical transition have been studied and showing both universal or trans-specific factors. Studies show that sexual healthcare setting is a 'especially exposed situation' and that healthcare provider's knowledge and attitude is of primary importance in terms of sexual health. ${ }^{1516}$

In the face of HIV threat, Pre-Exposure Prophylaxis (PrEP) ${ }^{17-19}$ has emerged as a promising strategy for most exposed populations. There are indications that this may also be the case for transgender people. ${ }^{20}$ However, the available evidence suggests that the use of PrEP is lower among this group. Among transmasculine individuals, international diffusion of PrEP has been accompanied by some research. ${ }^{20-28}$ In France, however, data are lacking.

A survey with 200 transgender individuals done by OUTrans, a community-based transgender organisation in France, found that 25\% of them do not know their HIV status. Also, that PrEP is little known and surrounded by a feeling of maladjustment. ${ }^{29}{ }^{30}$ In addition, screening for other STIs and particularly human papillomavirus infection (HPV, is under-practiced among transmasculine individuals. ${ }^{31}$ The low uptake of screening and low interest in PrEP contribute to the epidemic, maybe due to maladaptation or difficulties in access. While there is a good level of knowledge of prevention means among transmasculine people in France, the existing methods are perceived by trans people to be maladjusted. ${ }^{29}$ Regarding access, $33 \%$ of the OUTrans study sample felt that access to prevention methods was complicated. ${ }^{29}$

Another explanation for the underuse of these prevention methods may lie in the low risk perception among transgender people. ${ }^{21}$ Risk perception is a key step in establishing a prevention strategy. A study conducted in the USA shows that half of the transgender men diagnosed with HIV did not identify or report a potential HIV exposure. ${ }^{8}$ In addition to problems of accessibility and adaptation to prevention methods, risk perception appears to be a critical issue in understanding the determinants of transgender people's sexual health. This population group may not have access to the same resources for sexual health information as cisgender (ie, non-transgender) individuals, ${ }^{8}$ which may make more difficult for them to adapt their prevention strategies.

Transmasculine people are often erroneously considered to be only heterosexual and dating cisgender women with low risk of infection. ${ }^{32} 33$ These presumptions do not allow to consider the possible variety of sexual and preventive practices among these individuals, nor their impact in terms of exposure to HIV and other STIs. Lowered opportunities for emotional and sexual encounters, that accompany transphobia, can make it difficult to negotiate prevention.

These health vulnerabilities are linked to social factors, mainly the stigmatisation and marginalisation of transgender people, ${ }^{34}$ and act as cross-cutting, from the social dynamics of sexual encounter, ${ }^{35}$ to the negotiation of 
means of prevention. These relational dynamics seems to be of primary importance and will be investigated during this project.

\section{STUDY AIMS AND HYPOTHESES}

This project has two main objectives:

1 To investigate the health status and sexuality of transmasculine people, in their relation to HIV and other STIs. In a comprehensive approach, the goal is to document and understand sexual behaviours in relation to the psychosocial context in which they are produced. This context is made up of different strata ${ }^{36}{ }^{37}$ : individual, social, legal, symbolic, which will be apprehended in order to precisely grasp the different positive and negative determinants of health of transmasculine individuals.

From this first main objective arise two secondary objectives:

1. document the specificities of transmasculine people in terms of living conditions and social relations.

2. investigate the diversity of sexualities of transmasculine people to apprehend their repertoire of sexual and preventive practices and the meanings associated with them. This will improve the understanding of the HIV epidemic dynamics in this population, allowing the drafting of recommendations for effective health education and health promotion strategies adapted to this group.

2 The second main objective, embedded within the community-based research framework, ${ }^{38-40}$ aims to strengthen the research skills available at OUTrans, a transgender community-based organisation located in Paris, France. This will be done through exchange of skills and expertise, targeting the use and production of scientifically valid data. OUTrans has a relevant and legitimate presence in the field of transmasculine health in France and is involved in field actions aimed at education and health promotion, as well as in the setting up and running of workshops. It also produces data, notably through the creation, distribution and promotion of questionnaires for transmasculine people. However, currently OUTrans lacks the human and financial resources to carry out more complex research, which this project hopes to remedy.

Our hypotheses are:

Hypothesis 1: The normative framework (social norms, legislative framework, etc) affects the experience of transmasculine people. Negative factors such as transphobia, marginalisation and precariousness impact the health status of this group, with exposure to HIV, other STIs and poorer sexual quality of life being potential indicators of a pattern of domination and discrimination against transmasculine people. A better understanding of how this systemic logic impacts the health of transmasculine people will contribute to identify the hindrances and levers to good overall and sexual health.

Hypothesis 2: Sexual health depends, among other factors, on social representations related to sexuality, HIV and other STIs. Highlighting these representations should improve understanding of the HIV epidemic dynamics in the transmasculine population. Understanding the sexual health of transmasculine persons will, by extension, allow for a better understanding of the determinants of the overall health of this population.

\section{METHODS}

\section{Theoretical approach}

This research is in the field of social psychology and employs a community-based approach. ${ }^{3841}$ Our theoretical approach is inspired by the theory of social representations, ${ }^{42}{ }^{43}$ which are a form of common-sense knowledge, with a practical orientation that people use to adapt their behaviour. ${ }^{44-46}$ This approach will help us to comprehend the meanings that transmasculine individuals attribute to health and sexuality and how this unfolds as particular behaviours among this group. We furthermore mobilise a sociological background ${ }^{47-49}$ notably an intersectional framework $^{5051}$ in order to better grasp the various norms and power relationships that influence the intimacies of transmasculine individuals. Indeed, research shows that transgender people experience intersecting forms of marginalisation, which, among other issues, will result in worse health outcomes than those seen among cisgender individuals. A feminist intersectional approach will allow us to better understand how different layers of marginalisation, discrimination and health inequities interweave on the actual experience of transmasculine people but also how transmasculinities influence sexual health more specifically. ${ }^{52} 53$

\section{Study population}

Data collection will involve two distinct populations using purposive sampling. The first population consists in key informants about transmasculine health and sexuality. These are healthcare providers or community members who are both committed to accompany transgender people in their health path with dedicated services, whether it is a community-based organisation, or any other structure in the health and social field. This convenience sample of 12 interviewees will be composed with the help of OUTrans, with the possibility of being complement by consulting the French transgender community at large. Key informants will be identified with the criterion of being recognised by the transmasculine community as trustworthy and/or as professionals implementing good practices.

The second study population consists of transmasculine individuals. We plan to use a purposive sampling strategy using quotas. Quotas are based on different variables such as: age, gender identity, race, type of transition, disability and so on. This, in order to ensure a representation of different profiles in our sample, as recommended by our scientific board. We estimate interviewing up to 80 individuals, and will consider thematic saturation as a criterion for defining the final number of interviewees. ${ }^{54}$ Recruitment will be carried out throughout 
France through social media and/or advertising in places frequented by the community, depending on the sanitary circumstances and possible displacement restriction due to the COVID-19 pandemic. The large sample will strive to include a diversity of life paths ${ }^{55}$ particularly considering place of residence, self-identification, race, sexuality, age and class. They will be interviewed on their life paths, lives as transmasculine individuals, their health status and their sexualities.

All key informants or transmasculine respondents will be over 18 years old, will participate voluntarily with no monetary compensation. However, considering ethical recommendations, participation will be at no cost for participants, for instance transportation fees will be reimbursed. They will provide systematically an informed consent. We will guarantee the confidentiality of the interviews and the anonymity of the participants applying the best practices and the highest ethical standards (Transgender Research Informed Consent (TRICON) is available as a online supplemental file 2).

\section{Patient and public involvement}

This research uses a community-based approach that greatly involves the concerned people and professionals evolving around transgender health in the very making of the research design and analysis. The goal, in this design, is to attain balance in the participation of both parties, who are considered of equal though different expertise.

The study is accompanied by a steering committee that gathers in equal parts researchers and actors from the community (through the partner association) and the scientific board comprises researchers specialised in trans studies while some of them self-identify as trans themselves.

\section{Study design}

A community-based research requires that the people concerned are involved at every stage of the process. This research is co-coordinated by a research team in Social Psychology of Health based at the Université Lumière Lyon 2, and OUTrans Non-Governmental Organisation (NGO) based in Paris. The study is planned to last 2 years, from February 2021 to January 2023.

OUTrans was founded in April 2009 by trans people and for trans people. The organisation aims to: create a network of self-support between trans people; organise meetings and social events for trans people and/or people questioning their trans identity, but also between trans people and their relatives; develop actions to improve access to healthcare for transpeople; raise awareness about transidentity among the people with whom trans people deal during their transition (medical, social, educational, administrative, etc.); among other things.

University Lumière Lyon 2 team is recognised for its extensive experience in the social sciences and humanities investigation, and more particularly in the field of the social psychology of health. Community-based research is an important axe of the laboratory works, with projects such as GUNDO-SO ${ }^{56}$ or ANRS-IPERGAY ${ }^{57}$ being examples of this approach.

We will apply a triangulation strategy, ${ }^{58}$ operated both at epistemological level (social psychology, and community knowledge) and data collection procedures and analysis levels. We will triangulate data and analyses about the barriers and levers of transmasculine health. Concerning the studied populations, a data collection from key informants will be put in perspective of a more consequent data collection from the concerned persons. Different data collection techniques will be triangulated, notably individual interviews and focus groups to identify consensus and dissension. The focus groups will allow for a collective discussion of elements addressed from the interindividual perspective during the interviews. The focus groups will be spaces where the researchers will occupy a minor place, in order to leave all the space to the people concerned. Most importantly, we will apply a triangulation of perspectives at the time of the analyses between the subjectivities of the people of the research team, to cross the academic or associative views, the lived experiences, the cisgender and transgender points of view.

\section{Data collection techniques}

As part of the data will be collected during COVID-19 pandemic, remote data collection will be applied if necessary for both interviews and focus groups. Nevertheless, the original protocol submitted in September 2019 included face-to-face semistructured interviews, ${ }^{60} 61$ focus groups ${ }^{62}$ and field work notes. ${ }^{63}$ Interviews with key informants will be held from April to May 2021 and focus group on September 2021. Interviews with concerned people will be held from September 2021 to June 2022 followed by focus groups from June 2022 to August 2022.

Interviews and focus groups will be audio recorded with participants' consent and guided by semistructured scripts. The interview guide with key informants will focus on the following topics: professional path, positioning with regards to the medical and transgender communities, testimonies on good practices and care of transmasculine sexual health and their specific needs. The script for interviewing transmasculine individuals will cover themes related to health, sexuality, intimacy and HIV/ STIs prevention. The interview and focus group guides will be drafted in coordination with the partner NGO during the research, they will be provided when available in further publication of results. The interview guide use for the first data collection with key informants is available as a online supplemental file.

Subsequently, separate focus groups will be held with willing participants of both study population: one with key informants and four with transmasculine people. The focus groups allow participants to discuss the analyses of the interviews in a validating strategy, but it will also serve the community-based perspective in both providing additional scientific data and a space for participants to discuss and explore their struggles regarding sexual 
health as transmasculine individuals. The scripts will cover the following themes: preliminary analyses of the interviews, transmasculine health, intimacy, sexuality, life paths and coping strategies.

\section{Data analysis}

Audio-recorded data from interviews, focus groups and fieldnotes will be transcribed verbatim for analysis. Data will then be interpreted based on the theoretical frameworks that inform this research.

For the key informant interviews, we will conduct an inductive analysis. ${ }^{64}$ The aim will be to bring out the main themes by condensing the results. For this condensation stage, we will use a categorical thematic analysis. ${ }^{65}{ }^{66} \mathrm{We}$ will iteratively read all the transcripts to identifying the emerging themes. ${ }^{67}$ We will use the NVivo software to systematise our observations. ${ }^{68}$ Data will then be interpreted based on the theoretical frameworks that inform this research.

Regarding the interviews with transmasculine people, an initial interpretative phenomenological analysis (IPA) will help clarify the subjective logic of the interviewees. ${ }^{69} 70$ The IPA seeks to understand the logics of making sense of the experience of individuals in their production context. ${ }^{71}$ This type of analysis will be applied to different small batches of interviews, with a maximum of 10 participants. ${ }^{10}$ These distinct batches will be composed on the basis of certain sociodemographic link with our quotas or behavioural variables, allowing us to eventually draw a proximity of different profiles. We wish to conduct these analyses on different batches, in order to be able to account for the diversity of the interviewed population and to shed light on this aspect of the underrepresented population's diversity. In a second stage, we will apply a discursive analysis approach, namely Foucauldian discourse analysis, ${ }^{72}$ which allows us to question 'the relationship between discourse and the way individuals think or feel, how they live themselves (eg, their subjectivity), what they do (eg, their practices) and the social conditions in which these experiences take place'. ${ }^{73}$ This method appears relevant insofar as the issues of power and domination are at the heart of the analysis, as well as for eliciting social representations of health and prevention.

We will proceed a thematic analysis ${ }^{66}$ on focus groups ${ }^{74}$ with both populations.

\section{DISCUSSION}

This study will produce data specific to the transmasculine population and has been specifically designed to that end. This aims to overcome limitations on the knowledge about the transmasculine population that is produced as the by-product of studies designed for cisgender persons or other sexual identity or gender minorities. It will therefore help to capture as accurately as possible the living conditions, socioeconomic situations, concerns and positive determinants of health of transmasculine persons.
Health and sexual behaviours will be studied, documented and understood in their relationship with transness. Documenting the representations of health and sexuality among the transmasculine population will allow a better understanding of its relationship to sexual health and to HIV and other STIs prevention. This will also allow to analyse the adequacy of HIV prevention services in relation to the health needs identified among transmasculine individuals, which could contribute to the improvement of sexual health within this population. In line with our second objective, through methodological and financial support, this project will contribute to strengthening a community-based organisation of transgender people through research skills transference and its commitment to the production of scientifically reliable data. Potential positive outcomes of this process include the valorisation of OUTrans' activities, feeding its advocacy actions, and improving its abilities on funds raising.

This study may also bring a substantial contribution to building future studies concerning transmasculine people. The challenge would then be to form a cohort that allows for longitudinal observation and an understanding over the long term of the evolution of practices, functions and social representations associated with the sexuality of transmasculine people.

This study will presumably have limitations that should be addressed. Remote data collection due to the pandemic may limit participation for those with limited digital access. The community-based approach is an asset but also a possible bias in recruitment, since it will be influenced by the NGO setting and channels used to reach them, likely leading to an over-representation of young, more privileged and educated people with strong political beliefs. Harder to reach will be the older, more rural and precarious population.

\section{ETHICS AND DISSEMINATION}

FOREST protocol was approved by the Comité d'Évaluation Éthique (CEEI) de l'Institut National de la Santé Et de la Recherche Médicale (CEEI/International Review Board 00003888) on 15 September 2020. All participants will be invited to provided informed consent.

Results will be published in peer-reviewed journals and presented at scientific meetings, assuring transparency of data and anonymity of participants. To assure dissemination of findings to participants and communities, we will draft a report with key findings to disseminate through all communication channels available, whether more traditional (press, academia, institutional communication channels) or contemporary and widely used by the community (social networks such as Instagram or Tiktok). There will also be a video presenting some of the main results and that will sum up the research. Complementarily, informative flyers will be elaborated to communicate the study findings to participants and to key stakeholders to improve information, care and prevention considering overall and sexual health, HIV and STIs. 
In addition, data, results and protocol will be archived, as part of an open science approach, but also with the intention of historising and preserving an understudied yet vital area of research and making it accessible to the concerned and general population rather than to scientists only. This could also have the aim of facilitating access to quality information, and of relieving the burden on populations already highly solicited by the world of research. This can also be seen as a form of compensation and long-term valorisation of the time accorded without monetary compensation by the interviewees.

In terms of potential harms or increased vulnerability to the population which may occur from conducting or publishing results of this study could be that some misinterpretation of the results could essentialise or reinforce prejudices about transmasculine people (vulnerability, mental health issues). However, the fact that this research is coconducted by an association of transgender people and is accompanied by a scientific board that comprises people self-identifying as trans should ensure that the group will be particularly vigilant (because of their community, political and/or scientific involvement) of the potential instrumentalisation of this research. The coconstruction of the research in all its stages, including the preparation of the papers and other publication materials, seems to us the best safeguard to overcome any potential harm.

Acknowledgements We would like to express our warmest thanks to the members of the FOREST Scientific Board who provided general supervision. OUTrans NG0 members; Pallesi, Lucie; Pignedoli, Clark; Rivest, Paul. This project has benefited from support from the TGIR Huma-Num.

Contributors Members of the OUTrans NGO appear as "OUTrans NGO" in order to respect their wish for confidentiality. XM and SR-R contributed equally to this paper and are joint first authors. MX, OUTrans NGO and MP conceived and designed the FOREST protocol. XM, SR-R, OUTrans NGO drafted the work. DF and MP revised it critically. All the authors have made important intellectual contributions. FOREST Scientific Board: Baril, Alexandre; Beaubatie, Emmanuel; Del Río Carral, María; Dentella, Céline; Ferraz, Dulce; Ginouvès, Véronique; Mabire, Xavier; Mora, Marion; OUTrans NGO members; Pallesi, Lucie; Pignedoli, Clark; Préau, Marie; Rivest, Paul; Robin-Radier, Suzanne.

Funding This work is supported by the French agency ANRS I Maladies Infectieuses Émergentes, grant number 20365 FOREST. This work also benefited a support of the DILCRAH (Délégation Interministérielle à la Lutte Contre le Racisme, I'Antisémitisme et la Haine anti-LGBT) the French Interministerial Delegation for the Fight Against Racism, Antisemitism and Anti-LGBT Hatred.

Competing interests None declared.

Patient consent for publication Not applicable.

Provenance and peer review Not commissioned; externally peer reviewed.

Supplemental material This content has been supplied by the author(s). It has not been vetted by BMJ Publishing Group Limited (BMJ) and may not have been peer-reviewed. Any opinions or recommendations discussed are solely those of the author(s) and are not endorsed by BMJ. BMJ disclaims all liability and responsibility arising from any reliance placed on the content. Where the content includes any translated material, BMJ does not warrant the accuracy and reliability of the translations (including but not limited to local regulations, clinical guidelines, terminology, drug names and drug dosages), and is not responsible for any error and/or omissions arising from translation and adaptation or otherwise.

Open access This is an open access article distributed in accordance with the Creative Commons Attribution Non Commercial (CC BY-NC 4.0) license, which permits others to distribute, remix, adapt, build upon this work non-commercially, and license their derivative works on different terms, provided the original work is properly cited, appropriate credit is given, any changes made indicated, and the use is non-commercial. See: http://creativecommons.org/licenses/by-nc/4.0/.

\section{ORCID iDs}

Xavier Mabire http://orcid.org/0000-0001-9058-0085

Dulce Ferraz http://orcid.org/0000-0002-0443-3183

Marie Preau http://orcid.org/0000-0002-6239-1671

\section{REFERENCES}

1 Reisner SL, Poteat T, Keatley J, et al. Global health burden and needs of transgender populations: a review. Lancet 2016;388:412-36.

2 Baral SD, Poteat T, Strömdahl S, et al. Worldwide burden of HIV in transgender women: a systematic review and meta-analysis. Lancet Infect Dis 2013;13:214-22.

3 Kaplan RL, Sevelius J, Ribeiro K. In the name of brevity: the problem with binary HIV risk categories. Glob Public Health 2016;11:824-34.

4 Scheim A, Kacholia V, Logie C, et al. Health of transgender men in low-income and middle-income countries: a scoping review. BMJ Glob Health 2020;5:e003471.

5 Giami A, Beaubatie E. Gender identification and sex reassignment surgery in the trans population: a survey study in France. Arch Sex Behav 2014;43:1491-501.

6 Giami A, Le Bail J. Infection VIH et IST dans la population" trans": une revue critique de la littérature internationale, 2010. Available: https://halshs.archives-ouvertes.fr/halshs-00594941/ [Accessed 8 Sep 2017].

7 Bauer GR, Zong X, Scheim Al, et al. Factors impacting transgender patients' discomfort with their family physicians: a RespondentDriven sampling survey. PLoS One 2015;10:e0145046.

8 Centers for Disease Control and Prevention. Hiv and transgender people, 2019. Available: https://www.cdc.gov/hiv/group/gender/ transgender/index.html [Accessed 3 Aug 2019].

9 Safer JD, Tangpricha V. Care of the transgender patient. Ann Intern Med 2019;171:ITC1.

10 Martin TK, Coolhart D. "Because your dysphoria gets in the way of you...it affects everything." The mental, physical, and relational aspects of navigating body dysphoria and sex for trans masculine people. Sex Relatsh Ther 2019:1-18.

11 Thurston MD, Allan S. Sexuality and sexual experiences during gender transition: a thematic synthesis. Clin Psychol Rev 2018;66:39-50.

12 Anzani A, Lindley L, Prunas A, et al. "I Use All the Parts I'm Given ": A Qualitative Investigation of Trans Masculine and Nonbinary Individuals' Use of Body during Sex. Int J Sex Health 2021;33:58-75.

13 Lindley L, Anzani A, Prunas A, et al. Sexual satisfaction in trans masculine and Nonbinary individuals: a qualitative investigation. $J$ Sex Res 2021;58:222-34.

14 Lindley L, Anzani A, Galupo MP. What constitutes sexual Dissatisfaction for trans masculine and Nonbinary individuals: a qualitative study. J Sex Marital Ther 2020;46:612-29.

15 Asklöv K, Ekenger R, Berterö C. Transmasculine Persons' Experiences of Encounters with Health Care Professionals Within Reproductive, Perinatal, and Sexual Health in Sweden: A Qualitative Interview Study. Transgend Health 2021;21.

16 Pulice-Farrow L, Gonzalez KA, Lindley L. 'None of my providers have the slightest clue what to do with me': Transmasculine individuals' experiences with gynecological healthcare providers. Int $J$ Transgend Health 2020;31:1-30.

17 Grant RM, Lama JR, Anderson PL, et al. Preexposure chemoprophylaxis for HIV prevention in men who have sex with men. N Engl J Med 2010;363:2587-99.

18 McCormack SM, Noseda V, Molina J-M. PrEP in Europe - expectations, opportunities and barriers. J Int AIDS Soc 2016;19:21103.

19 Molina J-M, Capitant C, Spire B, et al. On-Demand preexposure prophylaxis in men at high risk for HIV-1 infection. N Engl J Med 2015;373:2237-46.

20 Sevelius JM, Deutsch MB, Grant R. The future of PreP among transgender women: the critical role of gender affirmation in research and clinical practices. J Int AIDS Soc 2016;19:21105.

21 Deutsch MB, Glidden DV, Sevelius J, et al. Hiv pre-exposure prophylaxis in transgender women: a subgroup analysis of the iPrEx trial. Lancet HIV 2015;2:e512-9.

22 Escudero DJ, Kerr T, Operario D, et al. Inclusion of trans women in pre-exposure prophylaxis trials: a review. AIDS Care 2015;27:637-41.

23 Klein A, Golub SA. Increasing access to pre-exposure prophylaxis among transgender women and Transfeminine Nonbinary individuals. AIDS Patient Care STDS 2019;33:262-9. 
24 Marquez S, Cahill S. Transgender Women and Pre-Exposure Prophylaxis : What We Know and What We Still Need to Know. National Center for Innovation in HIV care, 2015. Available: https:// www.avac.org/resource/transgender-women-and-pre-exposureprophylaxis-what-we-know-and-what-we-still-need-know

25 Marshall BDL, Mimiaga MJ. Uptake and effectiveness of PreP for transgender women. Lancet HIV 2015;2:e502-3.

26 Poteat T, Wirtz A, Malik M, et al. A gap between willingness and uptake: findings from mixed methods research on HIV prevention among black and Latina transgender women. J Acquir Immune Defic Syndr 2019;82:131-140.

27 Rael CT, Martinez M, Giguere R, et al. Barriers and facilitators to oral PreP use among transgender women in New York City. AIDS Behav 2018;22:1-10.

28 Rowniak S, Ong-Flaherty C, Selix N, et al. Attitudes, beliefs, and barriers to PreP among trans men. AIDS Educ Prev 2017;29:302-14.

29 OUTrans. La place de la médiation dans La Prévention Du VIH, des ist et des hépatites CheZ les personnes transmasculines, 2018.

30 OUTrans. Médiation et Prévention Du VIH, des ist et des hépatites CheZ les personnes transmasculines, 2018.

31 Hsiao KT. Screening for cervical cancer in transgender men | transgender care, 2016. Available: https://transcare.ucsf.edu/ guidelines/cervical-cancer [Accessed 3 Aug 2019].

32 Kenagy GP, Hsieh C-M. The risk less known: female-to-male transgender persons' vulnerability to HIV infection. AIDS Care 2005;17:195-207.

33 Reisner SL, Hughto JMW, Pardee DJ, et al. LifeSkills for men (LS4M): pilot evaluation of a Gender-Affirmative HIV and STI prevention intervention for young adult transgender men who have sex with men. J Urban Health 2016;93:189-205.

34 Gowar C, Azad Y. Trans people's access to HIV prevention, treatment and care services, 2019. Available: http://www.aidsimpact.com/ abstracts/-LZuMoORT99V9fz2xyRJ [Accessed 3 Aug 2019].

35 Dank M, Lachman P, Zweig JM, et al. Dating violence experiences of lesbian, gay, bisexual, and transgender youth. $J$ Youth Adolesc 2014:43:846-57.

36 Bronfenbrenner U. The ecology of human development: experiments by nature and design. Cambridge: Harvard University Press, 1979.

37 Doise W, Valentim JP. Levels of Analysis in Social Psychology. In: International Encyclopedia of the Social \& Behavioral Sciences. Oxford: Elsevier, 2015: 899-903. http://linkinghub.elsevier.com/ retrieve/pii/B9780080970868240324

38 Demange E, Henry E, Préau M, eds. From collaborative research to community-based research. A methodological toolkit. Paris: ANRS / Coalition Plus, 2012. http://www.coalitionplus.org/wordpress/wpcontent/uploads/2012/07/GUIDEgb.pdf

39 Israel BA, Schulz AJ, Parker EA, et al. Review of community-based research: assessing partnership approaches to improve public health. Annu Rev Public Health 1998;19:173-202.

40 Reece M, Dodge B. A study in sexual health applying the principles of community-based participatory research. Arch Sex Behav 2004;33:235-47.

41 Morin M, Terrade F, Préau M. Psychologie communautaire et psychologie de la santé : l'implication de la recherche psychosociale dans la promotion de la santé. Psychol Fr 2012;57:111-8.

$42 \mathrm{Joffe} H$. Risk: from perception to social representation. Br J Soc Psychol 2003;42:55-73.

43 Moscovici S. Social representations: explorations in social psychology. Gerard Duveen. New York, United States: New York University Press, 2000. http://eu.wiley.com/WileyCDA/WileyTitle/ productCd-0745622259.html

44 Bauer MW, Gaskell G. Towards a paradigm for research on social representations. J Theory Soc Behav 1999;29:163-86.

45 Lampropoulos D, Fonte D, Apostolidis T. Exploring the link between stigma and social representations among people with and without schizophrenia in the French context. Psychiatry Res 2019;272:595-601.

46 Morin M. Nouvelles définitions de la santé : un regard psychosocial. Spirale 2006;37:29-41

47 Dorlin E. Sexe, genre et sexualités: introduction la théorie féministe. Paris, France: Presses universitaires de France, 2008

48 Clair I. Faire Du terrain en féministe. Actes Rech En Sci Soc 2016;213:66-83.
49 Bereni L, Chauvin S, Jaunait A. Introduction aux gender studies: Manuel des études SUR Le genre. Bruxelles, Belgique: De Boeck, 2008.

50 Crenshaw K. Demarginalizing the intersection of race and sex: a black feminist critique of antidiscrimination doctrine, feminist theory, and antiracist politics. Fem Polit 1998:314-43.

51 Warner LR. A best practices guide to intersectional approaches in psychological research. Sex Roles 2008;59:454-63.

52 Van Schuylenbergh J, Motmans J, Coene G. Transgender and non-binary persons and sexual risk: a critical review of 10 years of research from a feminist intersectional perspective. Crit Soc Policy 2018;38:121-42.

53 Wesp LM, Malcoe LH, Elliott A, et al. Intersectionality research for transgender health justice: a Theory-Driven conceptual framework for structural analysis of transgender health inequities. Transgend Health 2019;4:287-96.

54 Saunders B, Sim J, Kingstone T, et al. Saturation in qualitative research: exploring its conceptualization and operationalization. Qual Quant 2018;52:1893-907.

55 Bertaux D, de Singly F. Le récit de vie. Paris, France: Armand Colin, 2016.

56 Bernier A, Yattassaye A, Beaulieu-Prévost D, et al. Empowering Malian women living with HIV regarding serostatus disclosure management: short-term effects of a community-based intervention. Patient Educ Couns 2018;101:248-55.

57 Puppo C, Mabire X, Cotte L, et al. Community-Based care in the ANRS-IPERGAY trial: the challenges of combination prevention. AIDS Educ Prev 2019;31:259-72.

58 Caillaud S, Doumergue M, Préau M, et al. The past and present of triangulation and social representations theory: a crossed history. Qual Res Psychol 2019;16:375-91.

59 Restivo L, Apostolidis T. Triangulating qualitative approaches within mixed methods designs: a theory-driven proposal based on a French research in social health psychology. Qual Res Psychol 2019;16:392-416.

60 Dicicco-Bloom B, Crabtree BF. The qualitative research interview. Med Educ 2006:40:314-21.

61 Brown JL, Vanable PA, Eriksen MD. Computer-Assisted self-interviews: a cost effectiveness analysis. Behav Res Methods 2008;40:1-7.

62 Marková I, Linell P, Grossen M, eds. Dialogue in focus groups: exploring socially shared knowledge. London, England: Equinox, 2007.

63 Stacey J. Can there be a feminist ethnography? Womens Stud Int Forum 1988;11:21-7.

64 Thomas DR. A general inductive approach for analyzing qualitative evaluation data. Am J Eval 2006;27:237-46.

65 Bardin L. L'analyse de contenu. Presses Universitaires de France, 1989.

66 Braun V, Clarke V. Using thematic analysis in psychology. Qual Res Psychol 2006;3:77-101.

67 Roberts K, Dowell A, Nie J-B. Attempting rigour and replicability in thematic analysis of qualitative research data; a case study of codebook development. BMC Med Res Methodol 2019;19:66.

68 Bazeley P, Jackson K. Qualitative Data Analysis with NVivo. Second edition. London, England: SAGE Publications, 2013. http://public. eblib.com/choice/PublicFullRecord.aspx? $\mathrm{p}=1187248$

69 Biggerstaff D, Thompson AR. Interpretative phenomenological analysis (ipa): a qualitative methodology of choice in healthcare research. Qual Res Psychol 2008;5:214-24.

70 Smith JA. Reflecting on the development of interpretative phenomenological analysis and its contribution to qualitative research in psychology. Qual Res Psychol 2004;1:39-54.

71 Haas V, Jodelet D. Pensée et mémoire sociales. In: Psychologie Sociale, 2007: 111-60.

72 Willig C. Introducing qualitative research in psychology. 3rd Edn. Maidenhead, England: Open University Press, 2013.

73 Willig C. L'analyse discursive. In: Les méthodes qualitatives en psychologie et sciences humaines de la santé. Malakoff, France: Dunod, 2017: 84-105.

74 Preau M, Doumergue M, Protiere C, et al. Acceptability of HIV curerelated trials: the challenges for physicians and people living with HIV (ANRS-APSEC). AIDS Care 2018;30:914-20. 